\title{
Foucault como filósofo de la educación: un balance crítico de sus contribuciones ${ }^{1}$
}

\author{
Tuillang Yuing Alfaro ${ }^{2}$
}

Recibido: 5 de julio de 2019 . Aceptado: 20 de agosto de 2019

\begin{abstract}
Resumen
El trabajo ensaya un balance de las contribuciones de la filosofía de Michel Foucault al dominio educativo, haciendo especial énfasis en la recepción iberoamericana de la cuestión. Con este fin desarrolla, en primer lugar, una referencia a la versatilidad disciplinaria del autor, así como sus opciones metodológicas. En segundo lugar, se refiere a la genealogía como lineamiento procedimental que ha permitido a diferentes autores elaborar análisis críticos al sistema educativo, mayoritariamente desde la sociología y la teoría social. Posteriormente se elaboran algunas notas de conjuntos que avanzan en los aportes de otros conceptos de Foucault para enriquecer estos análisis, como "biopolítica" y "gubernamentalidad". Finalmente, se señalan algunas conclusiones en las que se vislumbran ciertos aportes que, desde la filosofía, buscan aproximaciones de tipo propositivo a los modos de implementar lo educativo.
\end{abstract}

Palabras clave: Foucault - educación - disciplina - sociología de la educación - filosofía

\section{Foucault as a philosopher of education: a critical balance of his contributions}

\section{Summary}

This work seeks to gauge the philosophical contributions of Michel Foucault to the field of education, playing particular emphasis on how the issue has been addressed in Ibero-America. It therefore begins with a discussion of the writer's disciplinary versatility, as well as his methodological

1 Este trabajo forma parte del proyecto FONDECYT Iniciación n 11180085: “Biopolítica y racionalidad gubernamental: elementos para una renovación de los aportes de Foucault a la educación".

2 Chileno, Doctor en Filosofía. Profesor en la Facultad de Pedagogía. Academia de Humanismo Cristiano. Contacto: tuillangyuing@gmail.com.

ORCID: https://orcid.org/0000-0002-5028-377X 
options. It then examines genealogy as a procedural guideline that has allowed a number of authors to conduct critical analysis into the education system, mainly from a perspective of sociology and social theory. It goes on to present certain notes on works that provide advances in other concepts of Foucault to enrich these analyses, such as "biopolitics" and "governmentality". Finally, a number of conclusions are presented, showing contributions that in philosophical terms seek approaches of the proactive type to means of implementing educational actions.

Keywords: Foucault - education - discipline - sociology of education philosophy

\title{
Foucault como filósofo da educação: uma avaliação crítica de suas contribuições
}

\begin{abstract}
Resumo
O trabalho apresenta uma avaliação das contribuições da filosofia de Michael Foucault para o domínio educativo, enfatizando especialmente a recepção ibero-americana da questão. Com esta finalidade desenvolve, em primeiro lugar, uma referência à versatilidade disciplinar do autor, assim como suas opções metodológicas. Em segundo lugar, refere-se a genealogia como diretriz processual que permitiu a diferentes autores elaborar análises críticas do sistema educativo, principalmente a partir da sociologia e da teoria social. Posteriormente são elaboradas algumas notas de conjuntos que avançam nos aportes de outros conceitos de Foucault para enriquecer estas análises, como "biopolítica" e "governamentalidade". Finalmente, são apontadas algumas conclusões nas quais se visualizam certas contribuições que, desde a filosofia, buscam aproximações do tipo propositivo aos modos de implementar o educativo.
\end{abstract}

Palavras-chave: Foucault - educação - disciplina - sociologia da educação - filosofia

\section{Antecedentes generales}

Es Georges Canguilhem —-tal vez el más relevante de los epistemólogos de las ciencias de la vida - quien, haciendo gala de su formación hibrida entre filosofía y medicina, señala ya en la introducción a Lo normal y lo patológico que "La filosofía es una reflexión para la cual toda materia extranjera es buena, y, estaríamos dispuestos a decir, para la cual toda buena materia tiene que ser extranjera" (Canguilhem 
1971 11) Esta infidelidad declarada hacia una extranjeridad respecto de la filosofía tiene mucho que ver con un alejamiento de aquello que convencionalmente se ha denominado historia de la filosofía, entendida esta como un catálogo de autores consagrados e hilvanados bajo una suerte de serialidad cómoda, e incluso, a veces progresiva. Para Canguilhem, al contrario, una buena materia para la reflexión consiste precisamente en aquello que deteriora la comodidad e invita al pensamiento a la aventura y a disputar sus contratos de sentido: extraviarse en aquellos distritos inexplorados, tanto por su lejanía como por su cercanía.

Con todo, esta invitación parece haber sido recogida por su discípulo más célebre: Michel Foucault. En efecto, una de las características de su trabajo es, sin duda, establecer una suerte de borroneo de los límites disciplinarios. Lejos de conservar una fidelidad al canon filosófico, su gesto reflexivo apuesta a traspasar con atrevimiento la rigidez propia de las fronteras de la experticia y de los territorios académicos. Foucault así lo expresa en la introducción al Uso de los placeres, uno de los últimos textos que el autor destinó a publicación el año de su muerte. Foucault sostiene:

¿qué es la filosofía hoy — quiero decir la actividad filosófica— sino el trabajo crítico del pensamiento sobre sí mismo? ¿Y si no consiste, en vez de legitimar lo que ya se sabe, en emprender el saber cómo y hasta dónde sería posible pensar distinto? Siempre hay algo de irrisorio en el discurso filosófico cuando, desde el exterior, quiere orientar a los demás, decirles dónde está su verdad y cómo encontrarla, o cuando se siente con fuerza para instruirles proceso con positividad ingenua; pero es su derecho explorar lo que, en su propio pensamiento, puede ser cambiado mediante el ejercicio que hace de un saber que le es extraño (2010 12).

Un saber extraño que, en su propia consistencia, arremete contra el pensamiento de forma crítica, no para devolverle el protagonismo y la arrogancia de la afirmación tajante, sino para capturarlo en su movilidad, en su ligereza vociferante. 
Pues bien, de alguna manera, quisiéramos hacernos eco de esa extrañeza e intentar llevar a Foucault hacia un terreno del que, si bien no es totalmente ajeno, constituye no obstante una cuestión que el autor no plantea de modo decidido: nos referimos a la educación. Pretendemos entonces, ensayar algunas notas de conjunto sobre los aportes que, con y desde Foucault, es posible hacer al terreno de la educación, asumiendo la complejidad de este vocablo y las embrolladas tramas a las que se subscribe.

Así las cosas, esta articulación entre el trabajo de Foucault y aquello que, de manera un poco tosca, se adjetiva como el terreno educativo, no es de ninguna manera una cuestión transparente. Por ejemplo, François Dubet (2014) enfatiza la ausencia de un análisis dilatado sobre la institución escolar. Este autor pone de relieve la ligereza de los pronunciamientos de Foucault en relación a la escuela, en el mismo momento en que esta pareciera tener un rol protagónico en la conformación de la sociedad disciplinaria tal y como se describe en Vigilar y castigar (1998). De este modo, en este ir y venir de la prisión a la fábrica, al hospital y a la escuela, esta última queda extraviada en un perfil institucional que está lejos de atender a su especificidad. Con todo, para Dubet, no se trata tanto de acusar de mutismo al mismo Foucault como de subrayar cierta inespecificidad que se arrastra del paradigma disciplinario para comprender la cuestión escolar. Un déficit o carencia que los analistas hispanohablantes de Foucault habrían advertido (Varela 2009).

En una vereda radicalmente opuesta, hay autores que han incursionado en el funcionamiento de los dispositivos disciplinarios en el ámbito escolar, teniendo a Foucault como una referencia central. Así, por ejemplo, Keith Hoskin (1997) llega a proponer una clave de lectura de buena parte de la obra del francés a la luz del fenómeno educativo, entendido como un contrato de sentido entre las dimensiones del saber y el poder. Para Hoskin, Foucault, "Durante todo el tiempo realizó un análisis educativo, aunque pensara que se refería al poder o al saber" (33). Desde luego, a la luz de estos análisis, la pertinencia de Foucault en el ámbito de la educación se ha vuelto más explícita: "Foucault descubrió algo muy sencillo (aunque muy raro, sin em- 
bargo): el carácter central de la educación en la construcción de la modernidad" (Hoskin, en Ball 1997).

Es más, Hoskin llega a sugerir que Foucault es un "criptoteórico de la educación", apelando al doble sentido del vocablo "disciplina": según esta matriz educativa, la disciplina alude al doble trabajo que se ejerce sobre el discípulo: de una parte, presentarle y ofrecerle un saber, y, de otra, mantenerlo en cierta actitud frente a ese saber. En definitiva, para Hoskin, la gran herencia de Foucault habría sido una serie de interrogantes y problemas en torno a la historia y la tradición educativa.

Nuestra tarea será, entonces, la de ensayar parcialmente algunas notas de síntesis en torno a estas posiciones e intentar aproximarnos a los efectos y alcances que efectivamente puede tener la obra de Foucault en el terreno educativo. Con todo, para efectos de cumplir esta tarea, privilegiaremos la lectura y el uso que se ha hecho de esta cuestión en el ámbito iberoamericano, teniendo así un recorte bibliográfico abordable por nuestro empeño.

\section{Foucault y la educación: la genealogía como perspectiva metodológica}

Vale la pena, entonces, ensayar un balance, aun cuando sea de manera parcial. En primer lugar, es preciso insistir en esta suerte de anomalía que presenta el trabajo de Foucault cuando se intenta inscribir como un trabajo filosófico. Contra aquel sesgo que restringe la filosofía a la conformación de un canon de problemas y a un catálogo de autores, Foucault resulta un permanente punto de fuga. En efecto, su trabajo desarrolló una variedad de estrategias metodológicas adecuadas al carácter específico de investigaciones histórico-críticas sobre temáticas diversas, en las que la versatilidad para pensar y analizar problemas excede el dominio estricto de una disciplina para ir al encuentro de ámbitos culturales heterogéneos (Revel 2003 Veyne 1996 2008). Tal vez esta promiscuidad intelectual ha generado una amplitud de registros, de modo tal que los aportes de Foucault a la educación han sido recogidos y profundizados mayoritariamente desde la sociología 
y la teoría crítica. Deberemos revisar el modo como se ha conformado esta trayectoria dentro del paisaje intelectual hispanohablante.

En efecto, si la sociología se ha valido de los aportes de Foucault para dirigirlos al terreno de la educación, ello ha sido a partir de la perspectiva genealógica. En el caso de Foucault, esta aproximación surge al observar que toda conformación del saber está guiada por una cierta regularidad externa, por un cierto "orden del discurso" (Foucault 1971), que no es otra dimensión que la de las relaciones de poder, entendida como una variable que permite complementar y explicar el devenir de los saberes al interior de la historia, en conjugación con su materialidad institucional. Así, por ejemplo, el trabajo de Julia Varela y Fernando Álvarez-Uría, buscando elaborar una arqueología de la escuela, hacen de la genealogía la bandera que impulsa un tipo determinado de historia:

Se pretende, por el contrario, aplicar el método genealógico para abordar el pasado desde una perspectiva que nos ayude a descifrar el presente, a rastrear continuidades oscuras por su misma inmediatez, y a determinar los procesos de montaje de las piezas maestras, sus engarces, para qué sirven y a quiénes, a qué sistemas de poder están ligadas, cómo se transforman y disfrazan, cómo contribuyen, en fin, a hacer posibles nuestras condiciones actuales de existencia (Varela y Álvarez-Uría 1992 15).

De este modo, la genealogía consiste en un modo de interrogación retrospectivo, que busca explicar las condiciones históricas de existencia desde la perspectiva de las relaciones de fuerza y poder que sostienen y fundan prácticas sociales e institucionales (Morey 1981). El análisis genealógico permite mostrar que aquel sujeto antropológico, que está a la base de la configuración y desarrollo de las ciencias humanas y sociales -entre ellas, la educación-, es un objeto de saber que está también, correlativamente, sujeto a relaciones de poder (Revel 2003).

Por esta razón, Foucault ha servido como una consistente herramienta sociológica para elaborar un análisis crítico de la educación, tanto en su dimensión discursiva como en su dimensión político-institucional. 
Y, si bien, como ya señalamos, el autor no dedicó ningún trabajo exclusivo al estudio del sistema escolar, sus indagaciones han servido de punto de referencia para iniciar algunos análisis que recogen algunas de sus categorías.

De esta manera, una de las más celebres nociones que Foucault pone en discusión desde mediados de los setenta, es la llamada "sociedad disciplinaria". Se trata de un enfoque que el autor desarrolla ampliamente en Vigilar y castigar (Foucault 1998). En esta obra se muestra, con énfasis, el parecido de familia que existe entre las cárceles, las fábricas, los hospitales y, por cierto, las escuelas. Todas estas instituciones tienen, en la base, una cierta tecnología de poder que posibilita la regulación de la corporalidad y el principio de producción de tareas individuales, así como también la posibilidad de producción de saber por medio de la información obtenida del individuo como célula de análisis y de control (Morey 1981 Veyne 1996 2008).

Desde esta perspectiva, la aplicación de Foucault al terreno de la educación ha llevado a sugerir un paralelo entre la conformación de un saber educativo - a saber, la pedagogía —, anclado este en diferentes aportes, tanto de la antropología filosófica como de una incipiente psicología del desarrollo, y la institucionalización de la escuela al alero de los principios del Estado moderno, que toman a la infancia como una de sus preocupaciones. Desde luego, al inscribirse plenamente dentro del paradigma disciplinario, la escuela revela una lógica institucional y una imbricación profunda con una regulación de los cuerpos y de las conductas, que invita a una serie de análisis que hasta ese momento se habían insinuado con cierta timidez.

En este sentido, las principales diferencias de la perspectiva abierta por Foucault respecto de otras teorías críticas sobre educación proporcionadas por la sociología — principalmente las de la correspondencia (Baudelot y Establet 1990 Bowles y Gintis 1981) y las de la reproducción (Bourdieu y Passeron 2004)_, radican en que Foucault rompe, de forma decidida, con una concepción estatista del poder, así como con una visión dicotómica de los conflictos sociales - lucha de clases, dominantes versus dominados - que hasta el momento había servido como esquema explicativo del funcionamiento de las 
instituciones en su conjunto, desconociendo las especificidades y los matices de las racionalidades particulares que las integran (Varela y Álvarez-Uría 1992). En general, las investigaciones asentadas en el paradigma de la correspondencia o de la reproducción acusan una decisiva determinación de la esfera económica por sobre la educativa. En todas ellas, las funciones de la educación reposan tanto en las exigencias del capitalismo occidental como en la división social del trabajo y, en definitiva, en el sistema general de explotación y dominio. De este modo, el sistema escolar mantiene y recompone la estratificación y escalafones de las fuerzas productivas en dos redes independientes, que van desde el trabajo manual hasta la administración política y gestional del modelo. Así lo sintetiza Julia Varela: "La Escuela transmite la cultura dominante en ambas redes de forma diferenciada y con desigual intensidad, lo que conduce a producir, por una parte, proletarios sometidos $y$, por otra, agentes activos de la ideología dominante" (2009).

La educación no sería entonces sino una herramienta de extensión de la división jerárquica de las clases sociales. Desde luego, Foucault, al igual que otros autores - como los ya mencionados Baudelot y R. Establet, Bowles y H. Gintis, o incluso Bourdieu y Passeron-, toma como punto de partida para sus análisis una concepción del terreno social como un espacio en el que existen relaciones de poder, en el que tienen lugar conflictos por la hegemonía social, pero donde, no obstante, la mecánica y la dirección de estos enfrentamientos está lejos de responder a una racionalidad o mecánica única, y requiere, por tanto, de una variedad de análisis circunscritos a escenarios históricos delimitados (Morey 1981 Varela y Álvarez-Uría 1992). Si bien Foucault comparte con estos enfoques la búsqueda de comprensión de las funciones manifiestas de la educación, su trabajo dirige también su ofensiva hacia aquellas funciones latentes que actúan en la escuela y la pedagogía como prácticas en sí mismas. Antes que detenerse en las contradicciones de clase, los aportes de Foucault liberan la posibilidad de subrayar los variados y heterogéneos conflictos que se dan cita en el sistema educativo y en la producción de saberes que le es solidaria, articulando de otro modo su conexión con la generalidad del sistema social. En consecuencia, para hacer una genealogía de la 
cuestión educativa, la institución no sería en ningún modo un punto de partida, sino una resultante y un efecto a explicar. De ahí que se busque comprender cómo las instituciones han llegado a establecerse en diálogo y comunicación con ciertas prácticas y saberes que las legitiman. Teniendo esto en consideración, la escuela es, según Foucault, uno de los espacios en el que se ensayan procedimientos y tecnologías que hacen posible la formación de una nueva anatomía del cuerpo, de una microfísica del poder que disciplina y gobierna las conductas cotidianas. Una institución que fomenta una singular representación de la moralidad y que contribuye simultáneamente, en ciertos episodios históricos, a la formación de un determinado saber sobre el hombre y a la configuración de identidades sociales y subjetividades favorables a regímenes de producción y de organización social (Ball 1997).

Por cierto, estos aportes abren la puerta para una perspectiva crítica respecto del fenómeno educativo. En ese sentido, un matiz importante consiste en que estos análisis críticos, sobre todo en cuanto son dirigidos a la institución escolar, no pueden superponerse estrictamente ni restringirse a los intereses o estrategias exclusivos de una clase social ni política. Dicho de otro modo: la crítica a las instituciones disciplinarias también interpela a los gobiernos y sistemas de inspiración revolucionaria o progresistas. En consecuencia, la tarea de hacer del ser humano un cuerpo dócil y un alma buena o normalizada no puede ser leída únicamente bajo la égida de la burguesía dominante, sino que también puede advertirse y denunciarse en aquellos sistemas que declararon, en su momento, no ser cómplices de las ambiciones del capitalismo.

\section{El efecto Foucault como crítica a la educación: síntesis generales}

Como ya hemos adelantado, existe una generosa producción que ha utilizado este prisma en el terreno educativo. Para nuestros propósitos, enfocaremos nuestro análisis en los aportes que han tenido lugar en la esfera iberoamericana. Entre estos aportes destaca el trabajo de 
Julia Varela y Fernando Álvarez-Uría (1992), el cual atiende a la conformación de la escuela europea y su lugar en el Estado moderno, apelando abiertamente a la genealogía foucaulteana para ensayar una "arqueología de la escuela", complementada con insumos del marxismo y de la historia social de la infancia (Ariès 1973).

En una óptica similar, también deben subrayarse las contribuciones de Mariano Narodowski (1994) —tanto en solitario como en compañía de Ricardo Baquero (1990)—, quienes, también al alero de la obra de Ariès, elaboran un diagnóstico sobre la relación de la infancia y la institución, así como un balance analítico de la intromisión de la psicología en el terreno educativo. Para estos autores, una pregunta esencial consiste en averiguar bajo qué condiciones el saber pedagógico ha buscado establecer normalidades al estipular y configurar su proceder bajo ideales deseables y sus opuestos fallidos. Y para esta tarea la arqueología foucaulteana de las ciencias humanas aparece como una referencia ineludible. Atendiendo a esta tendencia, no es aventurado subrayar la oscilación del saber pedagógico bajo el paradigma cientificista positivista que se perseguía para las ciencias humanas: el valor impositivo de la norma — su normatividadconstituye entonces su mismo instrumento de validación: lejos de reconocer la positividad de los hechos o del dato, la normatividad pedagógica incrusta su régimen de validación en la nobleza y depuración de las metas que busca consolidar (Baquero 2008). De este modo, aun cuando la pedagogía ha ido abandonando de modo paulatino el discurso de la descalificación y la segregación, lo que ha puesto en su lugar son formas sutiles de administración en torno a la norma. La organización y taxonomización de conductas y ritmos en función del rendimiento, y las expectativas, se han conformado a partir de distinciones que se pretenden amparadas en la objetividad, pero que presentan, de igual manera, algunos supuestos epistemológicos que convendría revisar. Baquero y Narodowsky lo señalan de la siguiente forma:

Se producen así formas de exclusión y de posterior inclusión. La pedagogía localiza y relocaliza, segrega e integra, posterga y actualiza la ocurrencia de fenómenos a la luz 
de su poder. Poder analítico, poder productivo; poder normalizador, capaz de determinar beneficios y perjuicios, alta calidad y baja calidad, normalidad y patología (1990 39).

Por cierto, Olga Lucía Zuluaga (2005), junto al Grupo de Historia de la Práctica Pedagógica en Colombia, ha generado una serie de investigaciones multidisciplinarias, con vistas a reconstruir la historia pedagógica de su país atendiendo a sus aspectos históricos, políticos e ideológicos. En todas estas contribuciones, la inspiración genealógica es también explícita y elocuente. En sintonía con este trabajo, nos permitimos destacar las investigaciones de Marcelo Caruso (en Zuluaga 2005), quien indica que una de las primeras operaciones de la disciplina es una funcionalización del espacio, coherente con la organización de una cuadrícula o tabla del saber, propia de la episteme clásica, en la que, según Foucault, emergen las ciencias humanas. Como es sabido, para Foucault, la ambición fundamental de la época clásica decía relación con la representación exhaustiva del mundo. La realidad debía tener un correlato en el mundo de la representación y, por tanto, en el de la palabra. En esa perspectiva, el conocimiento es, en todos los casos, una medición que la razón hace de la realidad, de la cual puede extraer nuevas y diferentes elaboraciones de un orden que explica y dibuja el acercamiento al cosmos. En este contexto, el saber tiene que ver con la posibilidad cierta de una mathesis universal, concebida esta como una "ciencia universal de la medida y del orden" (Foucault 2002 63) que expresa la relación del conocimiento con el orden total. Luego, para Caruso, la escuela se elabora precisamente como un sistema de clasificación y de orden, que despliega conjuntamente ámbitos de saber y de poder en torno a tipos ideales de normalidad.

Evidentemente, este balance está lejos de ser exhaustivo y caben además una serie de precisiones que no viene al caso, para estos propósitos, detallar. Con todo, intentando elaborar un sumario conceptual de los trabajos sobre educación de inspiración foucaulteana, se puede señalar que entregan valiosas miradas críticas a la cotidianeidad escolar como dispositivo de impronta disciplinaria. No obstante, tienden a privilegiar la pregunta por lo educativo desde el plano de lo institu- 
cional, sin conceder la demora necesaria al ámbito del saber, en el que los discursos pedagógicos tienen un rol relevante.

A lo anterior debemos sumar los aportes de Rodrigo Castro (2004 2005), quien entrega algunas aproximaciones que resultan iluminadoras, tanto para el examen de aspectos metodológicos del autor, como para conocer antecedentes históricos y sociales que inciden en la construcción de la pedagogía moderna.

\section{Desarrollos posteriores y conclusiones provisorias}

Con todo, cabe destacar que muchos de estos desarrollos han sido elaborados con un corpus restringido de la obra de Foucault, por lo que tampoco se advierte un desarrollo suficiente del vínculo entre disciplina, biopolítica y gubernamentalidad, categorías difundidas y presentadas mayoritariamente en los cursos impartidos por Foucault en el Collège de France, y que son de publicación, en general, posterior a las propuestas de los autores aquí mencionados. No es de extrañar entonces que, al menos en el contexto iberoamericano, buena parte de los trabajos elaborados a partir de Foucault y dirigidos a la educación tengan como referencia central las tesis de Vigilar y castigar, razón por la cual la escuela, como institución disciplinaria, queda descontextualizada de una consideración de las políticas educativas globales en los niveles de población y gobierno. De este modo, las tensiones entre derechos, libertades y mercado parecen a veces desatendidas. Son precisamente estas temáticas las que hoy es posible abordar gracias a la serie de cursos de Foucault, aparecidos desde fines de los noventa, cuya publicación acaba de finalizar.

No obstante, para ser justos, debemos mencionar los aportes del brasilero Alfredo Veiga-Neto y de la argentina Silvia Grinberg, quienes han dado algunos pasos iluminadores que conectan el trabajo de Foucault con aquellos esquemas de análisis posteriores a Vigilar y castigar. Así, Veiga-Neto ensaya algunas observaciones sobre el lugar de las instituciones educativas dentro del esquema de las políticas neoliberales. Apelando al aparato conceptual, principalmente de Seguridad, territorio 
y población (2006) y Nacimiento de la biopolítica (2008), el autor sostiene que la educación sería aquel estamento que modela la relación entre la "normación" disciplinaria y la normalización de la seguridad y las poblaciones (Foucault 2006) bajo la óptica gubernamental:

Es claro que la educación articula muchas cosas, pero es en esa articulación entre los individuos y las poblaciones - de las cuales esos individuos forman parte- que yo me intereso [...] Mientras que las disciplinas exigen instituciones donde actuar, la regulación de las poblaciones exige políticas que organicen sus acciones (Veiga-Neto 2013 2).

Además, para Veiga-Neto, es el uso de la norma el que articula el plano del individuo con el plano de la población o, dicho con otra nomenclatura, entre el ámbito de la disciplina y el ámbito de la biopolítica. Esto permite que el enfoque gubernamental de Foucault asome como una variable amplia, en la que concursan tanto medidas institucionales como tácticas de gestión de la población y políticas de ordenamiento de las conductas en espacios controlados de libertad.

Por su parte, Silvia Grinberg (2011) ha atendido al modo en que las instituciones y políticas educativas han avanzado hacia un gerenciamiento que establece un contrato de sentido con una heterogeneidad de los modos de vida, en virtud de los cuales la precarización se asimila a la posibilidad de cambiar y hacerse cargo de sí mismo. A lo anterior se suma una racionalidad de gobierno, que renuncia al control de la totalidad en favor de una fragmentación social que descuida aquellos segmentos sociales castigados por la miseria y la desorientación. Con un trabajo de registro y observación atento en contextos de pobreza extrema, la autora desenmascara aquel relato que hilvana "crisis" a "posibilidad y emprendimiento". Se trata, en este sentido, de tomar nota de los dispositivos pedagógicos como "una enorme maquinaria de producción de subjetividad, esto es de gobierno de la población" (Grinberg 2011 5). Bajo este escenario, la jerga que enaltece valores como la diversidad y la tolerancia, aparece como un bálsamo para el abandono de los sectores desfavorecidos bajo la gramática del esfuerzo personal y el emprendimiento autónomo: 
Así, en la lógica del gerenciamiento, la racionalidad de fragmentos define los términos del gobierno de la población que se presentan en la forma del no relato, de relatos fragmentados que arrojan a la población a la gestión de sí en una no tan sui generis ética de la responsabilidad individual (Grinberg 2011 6).

En suma, se trata de una aproximación que conecta, de manera sagaz, la escuela con aquellos dispositivos que no solo gobiernan, sino que producen modos de subjetividad en lo que se ha llamado las "nuevas sociedades de control". En ese sentido, trabajos como los de estos autores elaboran hipótesis que pueden aún reformularse y que abren perspectivas fértiles en el uso del pensamiento de Foucault hacia el terreno educativo.

Por todas estas razones, es posible considerar que, de modo general, los aportes a la educación basados en Foucault han privilegiado la aplicación de la noción de "disciplina" como categoría de análisis. En ese sentido, a nuestro juicio, es aún posible profundizar en dos dimensiones: por una parte, en el ámbito discursivo que proporciona la arqueología de las ciencias humanas, en consonancia con la cuestión de las tecnologías de poder de las que son deudoras; $y$, por otra, en una puesta en relación del enfoque disciplinario con la re-consideración que el mismo Foucault hizo de la cuestión del poder a la luz de las nociones de biopolítica, seguridad y gubernamentalidad, entre otras. Un análisis del distrito de lo educativo desde el enfoque de la población, así como una consideración de las relaciones entre gobierno y mercado que entrega la biopolítica, podría dar nuevo vigor a los elementos metodológicos que la obra de Foucault aporta a lo educativo, además de sugerir una lectura de conjunto y refinar la relación entre los distintos momentos de la obra del autor, para enfatizar otros ámbitos de indagación en la esfera de la educación.

Finalmente, al revisar este abanico de producciones y las tentativas que de ellas se desprenden, se puede advertir que, dado el acento intencionado en la cuestión del poder que implementa el enfoque genealógico, el trabajo de Foucault invita a realizar un diagnóstico 
crítico de las distintas dimensiones de la educación. Se trata, entonces, de un diagnóstico que guarda estatura de denuncia, que se empecina en mostrar con nitidez los efectos normalizadores y las racionalidades reguladoras de la teoría y la institución educativa. Cabe entonces preguntar si acaso existe, por el contrario, una dimensión propositiva en la perspectiva que, a partir de Foucault, se puede elaborar en torno a la educación: ¿es posible ensayar o al menos imaginar otra educación desde lo que Foucault nos hereda? Sin intentar responder esta pregunta de manera precipitada, nos parece que, desde la filosofía, se vislumbra un recorrido que hace de las prácticas educativas una instancia más abierta al azar y, por tanto, más propicia para inventivas y puntos de fuga.

La respuesta, esta vez, parece venir entonces del lado de la filosofía. Así, por ejemplo, el trabajo de Walter Kohan (2004 y 2008) expone los puntos centrales para una relación de carácter propositivo entre Foucault y la educación, que ya no captura la educación en los límites coercitivos de la institucionalidad disciplinaria, sino que señala indicios para una escuela generadora de autonomía, en la que el sujeto se conforma a partir del ejercicio de "pensar de otro modo" (Foucault 1984). Por cierto, este trabajo se cruza con los aportes de Rancière (2007) y su re-descubrimiento de la figura de Jacotot, para de este modo establecer un vínculo cruzado entre una educación emancipadora y una subjetividad que escapa de sus inercias y certezas. En la misma línea, destaca el trabajo de Jorge Larrosa en Escuela, poder y subjetivación (1995), quien ha mostrado la relevancia de los últimos trabajos de Foucault como elementos presentes en distintos estamentos de la cotidianeidad escolar y donde las llamadas "tecnologías del yo" son una puerta abierta a imaginar otro tipo de relación con el saber o, si se quiere, a saber de otro modo.

Como ya hemos indicado, este balance es meramente tentativo y no busca de ninguna manera constituirse en un parámetro resolutivo. Con todo, nos ha permitido bosquejar los elementos generales a partir de los cuales la diversa y heterogénea obra de Foucault se topa con el terreno de la educación, y sugerir, de paso, algunas líneas de trabajo que pueden seguir siendo investigadas. Se trata, ante todo, 
de tentativas que vale la pena seguir revisando y que pueden, sin duda, dar nuevas luces sobre todo al alero de sus diversas perspectivas metodológicas. En definitiva, creemos que aclarar y sistematizar los nexos entre las estrategias metodológicas de Foucault permite un enfoque de conjunto, que actualizaría la filosofía y teoría de la educación que toma como base la obra del autor para, de esta manera, contribuir a iluminar los debates educativos que tienen lugar en la actualidad.

\section{Bibliografía}

Ariès, Philippe. L'enfant et la vie familiale sous l'Ancien Régime. París: Editions du Seuil, 1973.

Ball, Stephen (Comp). Foucault y la educación. Disciplinas y saber. Madrid: Morata, 1997.

Baquero, Ricardo. "De las dificultades de predecir: educabilidad y fracaso escolar como categorías riesgosas", en Construyendo posibilidad, apropiación y sentido de la experiencia escolar. Argentina: Homo Sapiens, 2008.

Baquero, Ricardo y Narodowsky Mariano. "Normatividad y normalidad en Pedagogía", Alternativas, Revista del Centro de Producción Educativa, Buenos Aires 4/6 (1990): 35-46.

Baudelot, Christian y Establet, Roger. El nivel educativo sube. Madrid: Morata, 1990.

Bourdieu, Pierre y Passeron, Jean Claude. La reproducción. México: Siglo XXI editores, 2004.

Bowles, Samuel y Gintis, Herbert. La instrucción escolar en la América capitalista. Madrid: Siglo XXI, 1981.

Canguilhem, Georges. Lo normal y lo patológico. Argentina: Siglo XXI editores, 1971.

Castro, Rodrigo. "Foucault y el saber educativo", Revista Diálogos Educativos, Santiago 8-10 (2004-2005).

Dubet, François. "Foucault et l'école: une étrange absence", Revue Sciences Humaines 19, París, 2014.

Foucault, Michel. L'Ordre du discours. Paris: Gallimard, 1971. 
Foucault, Michel. Un diálogo sobre el poder y otras conversaciones. Madrid: Alianza editorial, 1981.

Foucault, Michel. Vigilar y castigar. Nacimiento de la prisión. Buenos Aires: Siglo XXI editores, 1998.

Foucault, Michel. Las palabras y las cosas. Una arqueología de las ciencias humanas. Argentina: Siglo XXI editores, 2002.

Foucault, Michel. Seguridad, territorio, población. Curso en el Collège de France 1977-1978. Buenos Aires: Fondo de Cultura Económica, 2006.

Foucault, Michel. Nacimiento de la Biopolítica. Curso en el Collège de France 1977-1978. Buenos Aires: Fondo de Cultura Económica, 2008.

Foucault, Michel. Historia de la sexualidad II. El Uso de los placeres. Madrid: Siglo XXI editores, 2010.

Grinberg, Silvia. "Gubernamentalidad y educación en tiempos de gerenciamiento. Reflexiones en torno de la experiencia de los dispositivos pedagógicos en contextos de extrema pobreza urbana", Actas del VIII Encuentro de Cátedras de Pedagogía de Universidades Nacionales Argentinas. Buenos Aires: Universidad Nacional de La Plata, 2011.

Hoskin, Keith. "Foucault a examen", en Stephen Ball (Comp). Foucault y la educación. Disciplinas y saber. Madrid: Morata, 1997.

Kohan, Walter. Infancia. Entre Filosofía y Educación. Barcelona: Laertes, 2004.

Kohan, Walter. Filosofía, la paradoja de aprender y enseñar. Buenos Aires: Libros del Zorzal, 2008.

Larrosa, Jorge (Editor). Escuela, poder y subjetivación. Madrid: La Piqueta, 1995.

Morey, Miguel. "Prólogo", en Michel Foucault, Un diálogo sobre el poder y otras conversaciones. Madrid: Alianza editorial, 1981.

Narodowski, Mariano. Infancia y poder. Buenos Aires: Aique, 1994.

Rancière, Jacques. El maestro ignorante. Cinco lecciones sobre la emancipación intelectual. Buenos Aires: Libros del Zorzal, 2007.

Revel, Judith. Michel Foucault, expériences de la pensèe. París: Borda, 2003.

Varela, Julia y Álvarez-Uría. Fernando. Arqueología de la escuela, Madrid: La Piqueta, 1992.

Varela, Julia. "Educación (Sociología de la) Algunos modelos críticos", en Román Reyes (Dir) UCM Diccionario Crítico de las Ciencias Sociales, 2009. Disponible en: https://webs.ucm.es/info/eurotheo/diccionario/E/educacion_sociologia.htm. Consultado el 9 de mayo de 2018. 
Veiga-Neto, Alfredo. "Biopolítica, normalización y educación", Pedagogía y Saberes 38. Universidad Pedagógica Nacional (2013): 83-91.

Veyne, Paul. Foucault. Sa pensée, sa personne. Paris: Éditions Albin Michel, 2008.

Veyne, Paul. Comment on écrit l'histoire. Paris: Éditions du Seuil, 1996.

Zuluaga, Olga. Foucault, la Pedagogía y la Educación: Pensar de Otro Modo. Colombia: Universidad Pedagógica Nacional, 2005. 